\title{
PORE-SIZE DISTRIBUTION IN A BIOMEMBRANE MODEL
}

\author{
F.W. WIEGEL \\ Department of Applied Physics, Twente University of Technology, Enschede, The Netherlands
}

\author{
Received 30 March 1976
}

\begin{abstract}
It is shown that spontaneous formation of pores occurs in a recently proposed exactly solvable model for a twodimensional lipid bilayer. The statistical distributions of number and size of these pores are calculated from first principles.
\end{abstract}

In a recent paper the author has suggested a model for a two-dimensional lipid bilayer in which both shortrange repulsive forces and long-range attractive forces play a role, and for which the equilibrium statistical mechanics could be worked out in detail [1]. In this model the hydrocarbon chains of the lipids are restricted to the bonds of a square lattice; the short-range repulsion between the chains is simulated by the constraint that two chains cannot intersect, and the long-range attraction between two chains separated by a distance $\left|x-x^{\prime}\right|$ derives from a potential of the form:

$V\left(x-x^{\prime}\right)=-\frac{1}{2} m \gamma \omega_{0} \exp \left(-\gamma\left|x-x^{\prime}\right|\right)$.

Here $\gamma^{-1}$ is the range of the attractive force, $\omega_{0}$ denotes a positive constant and $2 \mathrm{ml}$ denotes the thickness of the membrane. ( $V / 2$ equals the distance between nearest neighbours in the underlying square lattice.) The potential (1) represents the total attractive interaction between two chains (mainly van der Waals interactions), but for mathematical convenience it had to be assumed that this total interaction acts between the head groups of the lipids only.

In the present note we would like to point out that in thermal equilibrium under the influence of the heat motion small holes will form in this bilayer, and we shall calculate their statistical characteristics. The formation of these pores can be understood physically in the following way. If the bilayer is in a configuration in which in a certain region the lipids are packed very closely, the energy is very low, corresponding to a large Boltzmann factor. However, closely packed hydrocarbon chains do not have many available configurations due to steric hindrance, so that the corresponding entropy is small. On the other hand, the bilayer can gain entropy by assuming a configuration in which each lipid has more space available. This leads to an increase in the energy of the configuration. Because of the very large number of degrees of freedom the outcome of this competitive process is very hard to forecast unless one can calculate the contributions of the various parts of the bilayer's phase space exactly. When this is done it is found that the most probable configurations of the bilayer are those in which the lipids are locally packed in long stretches of relatively high density, separated by short holes. Hence the density fluctuations in the membrane are highly non-linear.

The statistical properties of the pores can be found from the representation of the grand canonical partition function as a Wiener integral:

$$
\begin{aligned}
& Z(z, \beta, L)=N^{-1} \int \exp \left\{-\frac{\underline{m}}{2 \beta \omega_{0} \gamma^{2}} \int_{0}^{L}\left(\frac{\mathrm{d} \phi}{\mathrm{d} x}\right)^{2} \mathrm{~d} x\right. \\
& \left.-m \int_{0}^{L} B(\phi) \mathrm{d} x\right\} d[\phi(x)] .
\end{aligned}
$$

Here $L$ denotes the length of the membrane, $N$ a normalization constant, $\beta=k_{\mathrm{B}} T$ with $k_{\mathrm{B}}$ denoting Boltzmann's constant and $T$ the absolute temperature and

$B(\phi)=\frac{\phi^{2}}{2 \beta \omega_{0}}-\frac{1}{2 l} \beta p_{0}\left(\zeta e^{\phi}\right)$.

The function $\beta p_{0}$ is the grand canonical partition function of the model without the attractive interaction, and $\zeta$ is related to the fugacity $z$ by $\zeta=z \exp \left(-\frac{1}{4} \beta \omega_{0} \gamma^{2}\right)$.

In [1] the Wiener integral (2) was calculated straightforwardly, which leads to the thermodynamic functions of the bilayer. It was found that the isotherm showed a phase transition at a density $\rho=\rho_{\mathrm{s}}(T)$. The 
existence of non-linear density fluctuations, and the statistical distribution of their number and size can be extracted from (2) by means of a method which has been discussed in some detail in a previous review [2]; we need to point out only the main steps involved:

(a) The most probable configurations of the bilayer correspond to fields $\bar{\phi}(x)$ which maximize the integrand of (2). These fields are equal to 0 or $\phi_{\mathrm{S}}$ in most of the bilayer, with rapid transitions in between. The value of $\phi_{\mathrm{S}}$ is determined as the largest root of the equation $B^{\prime}\left(\phi_{s}\right)=0$. The distance over which a maximizing field makes the transition from the value 0 to the value $\phi_{\mathrm{S}}$ is of the order of $c / \gamma$, where $c$ is a function of $z$ and $\beta$, but not of $\gamma$ and $m$. A maximizing field corresponds to a configuration in which the membrane consists of stretches of densily packed lipids, separated by pores.

(b) The contribution of a maximizing field $\bar{\phi}(x)$, and its immediate vicinity in function space, to the right hand side of (2) is evaluated. This contribution leads to the probability of a pore of a certain length to occur in the membrane.

'In this way it is found that, for $\rho$ near $\rho_{\mathrm{s}}$, the number of pores $\left(N_{\mathrm{p}}\right)$ in the membrane is given by the expression:

$\frac{N_{\mathrm{p}}}{L} \cong \frac{\gamma}{c} K^{2}\left[\left\{1-\exp \left(\frac{m c}{\gamma} B\left(\phi_{\mathrm{s}}\right)\right)\right\}^{2}+4 K^{2}\right]^{-1 / 2}$

$$
\left(\rho \approx \rho_{\mathrm{S}}\right) .
$$

Here $K=\exp \left(-m c^{\prime} / \gamma l\right)$, where $c^{\prime}$, like $c$, is a function of $z$ and $\beta$, but not of $\gamma$ and $m$. For lipid bilayers $m \gg$ 1 and $\gamma l \ll 1$, so $K \ll 1$ and the number of pores per unit length is very small. As $B\left(\phi_{\mathrm{s}}\right)>0$ for $\rho<\rho_{\mathrm{s}}$ and $B\left(\phi_{\mathrm{S}}\right)<0$ for $\rho>\rho_{\mathrm{s}}$, the number of pores drops off very sharply when $\rho$ moves away from $\rho_{\mathrm{s}}$ in either direction. The length $(D)$ of a pore is found to have an exponential distribution:
$\mathrm{p}(D)=\Delta^{-1} \exp (-D / \Delta)$

where the average pore-size $(\Delta)$ is given by:

$$
\begin{aligned}
\Delta & \cong \frac{c}{2 \gamma} K^{-2}\left[\exp \left(\frac{m c}{\gamma} B\left(\phi_{\mathrm{s}}\right)\right)-1\right. \\
& +\sqrt{\left.\left\{\exp \left(\frac{m c}{\gamma} B\left(\phi_{\mathrm{s}}\right)\right)-1\right\}^{2}+4 K^{2}\right]} \quad\left(\rho \approx \rho_{\mathrm{s}}\right)
\end{aligned}
$$

Hence the average pore-size is of the order of the range $\gamma^{-1}$ of the attractive forces for $\rho>\rho_{s}$, but increases dramatically if $\rho \downarrow \rho_{\mathrm{s}}$.

Whereas these pores are very small and infrequent for densities larger than $\rho_{\mathrm{s}}$, their size increases rapidly when the density approaches $\rho_{\mathrm{s}}$ from above, and they also become more numerous, thus leading to a sharp increase in the permeability of the membrane for the cell fluid and the substances dissolved in it. It is interesting to note that this variation of membrane permeability can also be accomplished for fixed values of temperature and density by varying the parameter $\omega_{0}$, which is a measure for the strength of the lipid-lipid attraction: a slight increase in $\omega_{0}$ will increase $\rho_{s}(T)$, which causes a rapid increase in the premeability of the membrane.

The author would like to thank Professor L.E.H. Trainor and Charles J. Lumsden of the Department of Physics at the University of Toronto for introducing him to some of the problems of membrane biophysics.

\section{References}

[1] F.W. Wiegel, J. Stat. Phys. 13 (1975) 515.

[2] F.W. Wiegel, Phys. Rep. 16C (1975), section 2.4 . 